\title{
ON THE ZEROS OF JACOBI POLYNOMIALS $P_{n}^{\left(\alpha_{n}, \beta_{n}\right)}(x)$ \\ BY \\ D. S. MOAK $^{1}$, E. B. SAFF $^{2}$ AND R. S. VARGA ${ }^{3}$
}

\begin{abstract}
If $r_{n}$ and $s_{n}$ denote, respectively, the smallest and largest zeros of the Jacobi polynomial $P_{n}^{\left(\alpha_{n}, \beta_{n}\right)}$, where $\alpha_{n}>1, \beta_{n}-1$, and if $\lim _{n \rightarrow \infty} \alpha_{n} /$ $\left(2 n+\alpha_{n}+\beta_{n}+1\right)=a$ and if $\lim _{n \rightarrow \infty} \beta_{n} /\left(2 n+\alpha_{n}+\beta_{n}+1\right)=b$, then the numbers $r_{a, b}$ and $s_{a, b}$ are determined where

$$
\lim _{n \rightarrow \infty} r_{n}=r_{a, b}, \quad \lim _{n \rightarrow \infty} s_{n}=s_{a, b} \text {. }
$$

Furthermore, the zeros of $\left\{P_{n}^{\left(\alpha_{n}, \beta_{n}\right)}(x)\right\}_{n=0}^{\infty}$ are dense in $\left[r_{a, b}, s_{a, b}\right]$.
\end{abstract}

While a great deal is known (see Szegö [2]) about the asymptotic behavior of the zeros of Jacobi polynomials $\left\{P_{n}^{(\alpha, \beta)}(x)\right\}_{n=0}^{\infty}$ for a fixed type $(\alpha, \beta)$, there do not appear in the literature results concerning the limiting behavior of zeros of sequences of Jacobi polynomials $\left\{P_{n}^{\left(\alpha_{n}, \beta_{n}\right)}(x)\right\}_{n=0}^{\infty}$ where $\alpha_{n}$ or $\beta_{n}$ (or both) are allowed to grow with $n$. Results on this latter problem have application to the study of incomplete polynomials, as is discussed in The sharpness of Lorentz's theorem on incomplete polynomials [1]. The present note is used in that paper (cf. [1, Lemma 3.4]), and is published separately here because of its independent interest.

Because the polynomials $P_{n}^{\left(\alpha_{n}, \beta_{n}\right)}(x), n=0,1,2, \ldots$, are in general not orthogonal on $[-1,1]$, our results are not as detailed as the known theorems for a fixed type $(\alpha, \beta)$. Of course, we do know that for $\alpha_{n}>-1, \beta_{n}>-1$ all the zeros of $P_{n}^{\left(\alpha_{n}, \beta_{n}\right)}(x)$ lie in the open interval $(-1,1)$ and, using the Sturm Comparison Theory, we can easily prove

THEOREM 1. Let $r_{n}$ and $s_{n}$ be, respectively, the smallest and largest zeros of the Jacobi polynomials $P_{n}^{\left(\alpha_{n}, \beta_{n}\right)}(x)$, where $\alpha_{n}>-1, \beta_{n}>-1$. Suppose that

$$
\lim _{n \rightarrow \infty} \frac{\alpha_{n}}{\left(2 n+\alpha_{n}+\beta_{n}\right)}=a \text { and } \lim _{n \rightarrow \infty} \frac{\beta_{n}}{\left(2 n+\alpha_{n}+\beta_{n}\right)}=b \text {, }
$$

Received by the editors September 20, 1977.

AMS (MOS) subject classifications (1970). Primary 33A65; Secondary 33A70.

Key words and phrases. Jacobi polynomials, Sturm Comparison Theorem.

${ }^{1}$ Research supported by NSF Grant MCS 75-06687-UW 144-G244.

${ }^{2}$ Research supported in part by the Air Force Office of Scientific Research under Grant AFOSR-74-2688.

${ }^{3}$ Research supported in part by the Air Force Office of Scientific Research under Grant AFOSR-74-2729 and by the Energy Research and Development Administration (ERDA) under Grant EY-76-S-02-2075. 
and set

$$
\begin{aligned}
& r_{a, b}:=b^{2}-a^{2}-\left[\left(a^{2}+b^{2}-1\right)^{2}-4 a^{2} b^{2}\right]^{1 / 2}, \\
& s_{a, b}:=b^{2}-a^{2}+\left[\left(a^{2}+b^{2}-1\right)^{2}-4 a^{2} b^{2}\right]^{1 / 2}
\end{aligned}
$$

Then,

$$
\lim _{n \rightarrow \infty} r_{n}=r_{a, b} \text { and } \lim _{n \rightarrow \infty} s_{n}=s_{a, b} .
$$

Furthermore, the zeros of the sequence $\left\{P_{n}^{\left(\alpha_{n}, \beta_{n}\right)}(x)\right\}_{n=0}^{\infty}$ are dense in the interval $\left[r_{a, b}, s_{a, b}\right]$.

The proof requires the following known results related to the Sturm Comparison Theory: (cf. Szegö [2, pp. 19-20]):

LEMMA 2. Let $H(\theta)$ be continuous on $\left(\theta_{1}, \theta_{2}\right)$ and suppose that $u(\theta)$ satisfies $u^{\prime \prime}+H(\theta) u=0$ for $\theta \in\left(\theta_{1}, \theta_{2}\right)$. If $H(\theta) \geqslant n>0$ on $\left(\theta_{1}, \theta_{2}\right)$, then $u(\theta)$ has $a$ zero in every subinterval of $\left(\theta_{1}, \theta_{2}\right)$ of length $\geqslant \pi / \sqrt{n}$.

LEMMA 3. Let $H(\theta)$ be continuous and negative in $\left(\theta_{1}, \theta_{2}\right)$. Then an arbitrary solution $u(\theta)(\not \equiv)$ of $u^{\prime \prime}+H(\theta) u=0$, for which $u(\theta) \rightarrow 0$ if $\theta \rightarrow \theta_{2}^{-}$, cannot vanish in $\theta_{1} \leqslant \theta<\theta_{2}$.

Proof of TheORem 1. As is known [2, p. 67], the function

$$
u_{n}(\theta):=\left(\sin \frac{\theta}{2}\right)^{\alpha_{n}+1 / 2}\left(\cos \frac{\theta}{2}\right)^{\beta_{n}+1 / 2} P_{n}^{\left(\alpha_{n}, \beta_{n}\right)}(\cos \theta)
$$

satisfies the differential equation

$$
d^{2} u / d \theta^{2}+H_{n}(\theta) u=0, \text { for } 0<\theta<\pi,
$$

where

$$
H_{n}(\theta):=\frac{1-4 \alpha_{n}^{2}}{16 \sin ^{2}(\theta / 2)}+\frac{1-4 \beta_{n}^{2}}{16 \cos ^{2}(\theta / 2)}+\left(n+\frac{\alpha_{n}+\beta_{n}+1}{2}\right)^{2} .
$$

It is convenient to rewrite $H_{n}(\theta)$ in the form

$$
\begin{aligned}
H_{n}(\theta)= & \frac{-\left(2 n+\alpha_{n}+\beta_{n}+1\right)^{2} \cos ^{2} \theta+2\left(\beta_{n}^{2}-\alpha_{n}^{2}\right) \cos \theta}{4\left(1-\cos ^{2} \theta\right)} \\
& +\frac{\left(2 n+\alpha_{n}+\beta_{n}+1\right)^{2}+1-2 \alpha_{n}^{2}-2 \beta_{n}^{2}}{4\left(1-\cos ^{2} \theta\right)}
\end{aligned}
$$

Notice that the numerator of $H_{n}(\theta)$ in (8) is, for $n \geqslant 1$, a quadratic in $x=\cos \theta$ having negative leading coefficient. The roots of this quadratic are: 


$$
\begin{aligned}
x_{n}^{ \pm}:= & \frac{\beta_{n}^{2}-\alpha_{n}^{2}}{\left(2 n+\alpha_{n}+\beta_{n}+1\right)^{2}} \\
& \pm\left[1+\frac{\left(\beta_{n}^{2}-\alpha_{n}^{2}\right)^{2}}{\left(2 n+\alpha_{n}+\beta_{n}+1\right)^{4}}+\frac{1-2 \alpha_{n}^{2}-2 \beta_{n}^{2}}{\left(2 n+\alpha_{n}+\beta_{n}+1\right)^{2}}\right]^{1 / 2} .
\end{aligned}
$$

Because $\alpha_{n}>-1$ and $\beta_{n}>-1$, then $2 n+\alpha_{n}+\beta_{n}+1>2 n-1>0$ for all $n \geqslant 1$, so that $\lim _{n \rightarrow \infty} 1 /\left(2 n+\alpha_{n}+\beta_{n}+1\right)^{2}=0$. Thus, with (1), the roots of (9) approach

$$
b^{2}-a^{2} \pm\left[1+\left(b^{2}-a^{2}\right)^{2}-2\left(a^{2}+b^{2}\right)\right]^{1 / 2},
$$

which are precisely the numbers $r_{a, b}$ and $s_{a, b}$ defined in (2) and (3). Since $\alpha_{n}>-1$ and $\beta_{n}>-1$, it easily follows from (1) that $a, b \in[0,1]$. Furthermore, from definitions (2) and (3), it can be verified that

$$
-1 \leqslant r_{a, b} \leqslant s_{a, b} \leqslant 1 \text {, }
$$

and that

$$
r_{a, b}=-1 \text { iff } b=0, s_{a, b}=1 \text { iff } a=0 .
$$

Returning to the differential equation (6), it follows from the above discussion that for each $\varepsilon>0$ sufficiently small,

$$
H_{n}(\theta)<0 \text { for } \cos \theta \in \begin{cases}\left(-1, r_{a, b}-\varepsilon\right), & \text { if } b>0, \\ \left(s_{a, b}+\varepsilon, 1\right), & \text { if } a>0,\end{cases}
$$

provided that $n$ is sufficiently large. Hence, by applying Lemma 3 to the function $u_{n}(\theta)$ in (5), we have $P_{n}^{\left(\alpha_{n}, \beta_{n}\right)}(x) \neq 0$ in $\left[-1, r_{a, b}-\varepsilon\right) \cup\left(s_{a, b}+\varepsilon, 1\right]$ for all $n$ large. In terms of the largest and smallest zeros of $P_{n}^{\left(\alpha_{n}, \beta_{n}\right)}(x)$, this means that

$$
r_{a, b}-\varepsilon \leqslant \liminf _{n \rightarrow \infty} r_{n}, \quad \limsup _{n \rightarrow \infty} s_{n} \leqslant s_{a, b}+\varepsilon,
$$

and letting $\varepsilon \rightarrow 0^{+}$yields

$$
r_{a, b} \leqslant \liminf _{n \rightarrow \infty} r_{n}, \quad \limsup _{n \rightarrow \infty} s_{n} \leqslant s_{a, b},
$$

the inequalities (13) being valid even if $a$ and/or $b$ are zero.

Next, we consider the inequality

$$
H_{n}(\theta) \geqslant n,
$$

which, using (8), is equivalent to

$$
\left(-A_{n} \cos ^{2} \theta+B_{n} \cos \theta+C_{n}\right) / 4\left(1-\cos ^{2} \theta\right) \geqslant 0,
$$

where

$$
\begin{aligned}
& A_{n}:=\left(2 n+\alpha_{n}+\beta_{n}+1\right)^{2}-4 n, \quad B_{n}:=2\left(\beta_{n}^{2}-\alpha_{n}^{2}\right), \\
& C_{n}:=\left(2 n+\alpha_{n}+\beta_{n}+1\right)^{2}+1-2 \alpha_{n}^{2}-2 \beta_{n}^{2}-4 n .
\end{aligned}
$$


It is immediately verified from (1) that the roots of the quadratic numerator in (15) again approach the number $r_{a, b}$ and $s_{a, b}$ as $n \rightarrow \infty$. Consequently, for each $\varepsilon>0$ sufficiently small,

$$
H_{n}(\theta) \geqslant n \text { for } \theta \in\left[\cos ^{-1}\left(s_{a, b}-\varepsilon\right), \cos ^{-1}\left(r_{a, b}+\varepsilon\right)\right],
$$

provided that $n$ is sufficiently large. Thus, by Lemma 2 , the function $u_{n}(\theta)$ has zeros within $\pi / \sqrt{n}$ of each of the endpoints of the interval in (17), and so

$$
\limsup _{n \rightarrow \infty} r_{n} \leqslant r_{a, b}+\varepsilon, \quad \liminf _{n \rightarrow \infty} s_{n} \geqslant s_{a, b}-\varepsilon .
$$

Letting $\varepsilon \rightarrow 0^{+}$and using (13) we have proved (4).

The fact that the zeros of the sequence $\left\{P_{n}^{\left(\alpha_{n}, \beta_{n}\right)}(x)\right\}_{n=0}^{\infty}$ are dense in $\left[r_{a, b}, s_{a, b}\right]$ also follows from Lemma 2 and the previous discussion.

As a special case of Theorem 1, we have

COROllaRY 1. If $\alpha$ and $\beta$ are finite such that $\lim _{n \rightarrow \infty} \alpha_{n} / n=\alpha$ and $\lim _{n \rightarrow \infty} \beta_{n} / n=\beta$, then the conclusions of Theorem 1 are valid with $a:=$ $\alpha /(2+\alpha+\beta)$ and with $b:=\beta /(2+\alpha+\beta)$.

We remark that Theorem 1 also includes cases where $\alpha_{n} / n \rightarrow+\infty$ and/or $\beta_{n} / n \rightarrow+\infty$.

\section{REFERENCES}

1. E. B. Saff and R. S. Varga, The sharpness of Lorentz's theorem on incomplete polynomials, Trans. Amer. Math. Soc. 249 (1979), 163-1 86.

2. G. Szegö, Orthogonal polynomials, Amer. Math. Soc. Colloq. Publ., vol. 23, Amer. Math. Soc., Providence, R. I., 1975.

Department of Mathiematics, University of Wisconsin, Madison, Wisconsin 53706

Department of Mathematics, University of South Florida, Tampa, Florida 33620

Department of Mathematics, Kent State University, Kent, OHo 44242 